\title{
EFFECTS OF ABUNDANCE CHANGES ON THE SPECTRA OF VERY COOL WHITE DWARFS
}

\author{
R. WEHRSE
}

Lehrstuhl für Theoretische Astrophysik, Universität Heidelberg F.R.G.

\begin{abstract}
In order to study the atmospheres of cool white dwarfs of spectral types DA, DI: and DG, model atmospheres have been calculated for temperatures $5000 \leqslant T_{\text {eff }} \leqslant 7000 \mathrm{~K}$ and gravities $7 \leqslant$ $\log g<8.5$. The helium to hydrogen abundance ratio ranges from 0.1 to 1000 . The abundances of the metals $\epsilon_{M}$ (relative to hydrogen) vary between the solar value $\epsilon_{M}^{\circ}$ and $\epsilon_{M}^{\circ} / 1000$. The models are hydrogen and metal line blanketed and in radiative equilibrium. The models with increased He abundance are characterized by a decreased mean opacity since the absorption cross-section of $\mathrm{He}$ is much smaller than that of $\mathrm{H}^{-}$. As a result, the spectral lines become stronger and - due to the much higher pressures - much broader. A reduction of the metal contents also increases the pressures. In this case it is caused by the shortage of electron suppliers. Here the spectral lines also become broader, but in general weaker.

These effects strengthen the overlap of the lines due to the high gravity of white dwarfs and more or less lead to a quasi-continuum which may be much below the true continuum. Because of the wavelength dependence of the line density the depression is strongest in the UV and blue spectral region, whereas for wavelengths $\lambda>4500 \AA$ the difference from the true continuum is only a few per cent. The details will be published elsewhere.
\end{abstract}

\section{DISCUSSION}

Kandel: In evaluating the electron depletion by formation of negative ions, did you take into account the pressure detachment of these negative ions?

Wehrse: Pressure ionization has been taken into account by means of a very simple hydrogenic approximation, i.e. in the case of negative ions it only enters via the cut-off of the partition function of the neutral species.

Querci: How many molecules have you introduced in your calculations?

Wehrse: $\mathrm{H}_{2}, \mathrm{H}_{2} \mathrm{O}, \mathrm{CH}, \mathrm{C}_{2}, \mathrm{CN}, \mathrm{CO}, \mathrm{CO}_{2}, \mathrm{NH}, \mathrm{NO}, \mathrm{N}_{2}, \mathrm{OH}, \mathrm{MgH}, \mathrm{CaH}$, and TiO have been taken into account. 